\title{
Social Robots as the Bride? Understanding the Construction of Gender in a Japanese Social Robot Product
}

\author{
Jindong $\operatorname{Liu}^{1}$ (D) \\ 1 School of Journalism and Communication, The Chinese University of Hong Kong, China
}

\begin{abstract}
This study critically investigates the construction of gender on a Japanese hologram animestyle social robot Azuma Hikari. By applying a mixed method merging the visual semiotic method and heterogeneous engineering approach in software studies, the signs in Azuma Hikari's anthropomorphized image and the interactivity enabled by the multimedia interface have been analyzed and discussed. The analysis revealed a stereotyped representation of a Japanese "ideal bride" who should be cute, sexy, comforting, good at housework, and subordinated to "Master"-like husband. Moreover, the device interface disciplines users to play the role of "wage earner" in the simulated marriage and reconstructs the gender relations in reality. It suggests the humanization of the objects is often associated with the dehumanization and objectification of the human in reverse.
\end{abstract}

Keywords: human-machine communication, social robots, gender representation, visual semiotic method, software studies

\section{Introduction}

The construction of the gender on media has been a major focus in cultural studies in the tradition of Birmingham school (Hall, 1996). Over the last decades, there have been a number of studies on media representation of the gender on a variety of media forms including television, newspapers, magazines, films, and social media platforms (e.g., Dobson,

CONTACT Jindong Liu (D) - School of Journalism and Communication - The Chinese University of Hong Kong • Humanities Building, Room 008, Shatin, N.T. • Hong Kong, China • jindongliu@link.cuhk.edu.hk 
2016; Hall, 1996; Ho, 2017; Lovdal, 1989; Toffoletti \& Thorpe, 2018). However, due to the fast-changing media technologies and landscape, current literature on the media representation of the gender has not adequately covered some new forms of media, such as anthropomorphized social robots.

With the rapid and disruptive development of Information and Communication Technologies (ICTs), especially Artificial Intelligence (AI) in recent years, there is a surge in the production of various types of anthropomorphized social robots, including not only traditional physical embodied machines but also virtual agents, such as chatbots and hologram social robots. Anthropomorphism is defined as the practices of attributing human characteristics into non-human entities, which is strongly associated with the essential concept of social robots (Duffy, 2003). With the natural human language processing and simulation of a human image, the logic of anthropomorphism is embedded in the production of social robots; whereas anthropomorphism is never neutral. From a critical perspective of cultural studies, it represents producers' ideologies and social values within particular cultural contexts, including their gender ideology (Gehl, 2014). Similarly, from the perspective of a feminist science and technology studies (STS), the increasingly humanlike machines also drive the modern feminist scholars such as Suchman (2008, p. 140) to embrace the "increasingly evident inseparability of subjects and objects, 'natural' bodies and 'artificial' augmentations," following the pace of Donna Haraway (2006) in seeking for the post-humanistic dissolving binary between males and females in her Cyborg Manifesto. Robertson (2010) suggests that anthropomorphized robots are "the vanguard of posthuman sexism" (p. 1). However, the biased representation of gender in social robots nowadays may not dissolve the gender binary, but instead reinforce it. As social robots are increasingly sexualized and entering our daily social interaction (Appel et al., 2019; Chambers, 2018; Scheutz \& Arnold, 2016), it is important to understand how gender ideology is represented and possibly influences people's understanding of gender in social robots.

Moreover, in social robots, the gender ideology may not only be represented through the media representation of social robots, but also be constructed dynamically. Different from previous media, social robots as a new form of digital media are distinguished in carrying its own agency to participate in the social interaction with human beings. Based on media equation theory (Reeves \& Nass, 1996), human users may treat them as equivalent human interlocutors that can not only "be" but also "do" things through the constant interactions between agents (Latour, 1996). As Butler (1988) suggests that women are not being but becoming, it is important to adapt a dynamic view to scrutinize the representation of gender, in a sense that it is one step further from representation, to the reconstruction of meanings. In this study, a general question we are interested in is: how is the meaning of gender represented and reconstructed dynamically on emerging social robot products?

To address this question, this study concentrates on a particular social robot product: a hologram device produced by a Japanese technology company named Gatebox Inc. (2020), which is inhabited by an original character anthropomorphized as a beautiful anime-style girl, named Azuma Hikari (see Figure 1). We chose this product because it is one of the earliest and probably most well-known hologram social robots attracting multiple international news reports (Bolton, 2016; Boxall, 2019; Gilbert, 2017). Gatebox (2020) announces the role of Azuma Hikari as a "comforting bride," which drives her human users into a simulated human relationship of marriage with her. Unlike other home devices that perform 
as an "assistant" (e.g., Alexa or Google Home), Azuma Hikari is designed to play a more intimacy-involved social role that is reconstructing the idea of gender in a simulated gender relation. Following the general question, a particular research question of this study is: How is gender represented and reconstructed dynamically on hologram social robot Azuma Hikari?

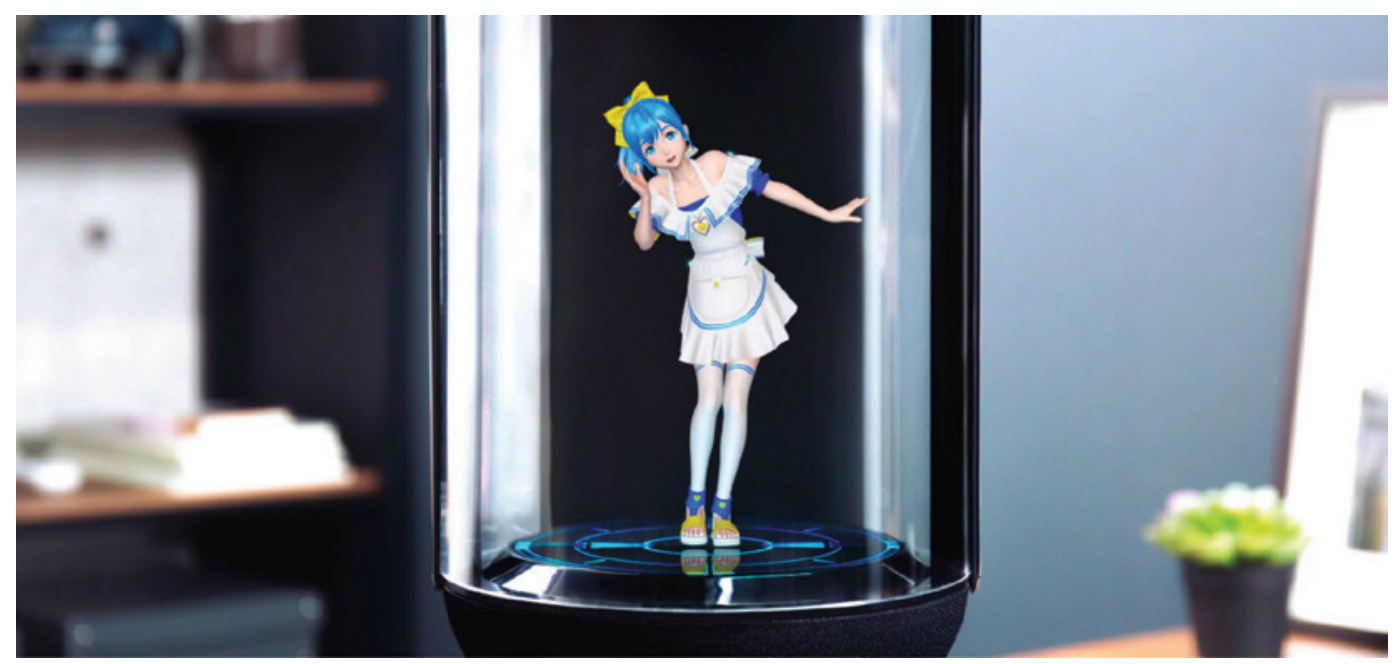

FIGURE 1 The Image of Azuma Hikari Shown on Gatebox's Website (Gatebox, 2020)

\section{Methods}

In response to the research question, this study applied the semiotic approach in toolkits of visual methods compiled by Rose (2016) and software studies approach in science and technology studies (STS) tradition. The former was chosen because it can help us understand the "being" of gender represented on the unique hologram visual materials of this new type of social robot that has rarely been studied before; the latter, on the other hand, provided a view for observing the "doing" of gender in the dynamic process of human-robot interaction. To be clarified, they were not separate methods but can be integrated into a continuous one in this study. As the hologram device in our case entails software techniques such as a multimedia interface that affords the strong interactivity in a visual sense, which requires us to forge a mixed approach to cover both.

\section{Visual Semiotic Method}

This study starts with visual methods to study the meanings of signs embedded in Azuma Hikari's image. By visual methods, it refers to a compilation of methods summarized by Rose (2016) for studying the visual materials. Rose classifies them into four sites of the visual: production, circulation, audiences, and the image itself. Due to the currently limited access to the other three sites, this study will primarily focus on the site of image itself. Among the interpretative approaches studying the site of image itself (p. 24), semiology has 
been seen by Rose (2016, p. 106) as the most prominent method to lead the discussion of the visual. It refers to the study of signs in the images, which are associated with how the meanings of the visual are created. Rose quoted historians Bal and Bryson (1991, p. 174 in Rose, 2016) to stress the importance of signs in semiology: "Human culture is made up of signs, each of which stands for something other than itself, and the people inhabiting culture busy themselves making sense of those signs (p. 107)."

In contrast to content analysis that justifies itself to be scientific based on its quantitative approaches, semiologists receive more formative influences from Marx, looking at the ideology beyond the "scientific knowledge." According to Rose (2016), "Ideology is knowledge that is constructed in such a way as to legitimate unequal social power relations; science, instead, is knowledge that reveals those inequalities (p. 107)." It is consistent with Eco (1980; Bianchi, 2015), who believes semiotic tools have the potential for grasping the interpretative dynamics of texts and discourse that carry the transmission of ideological meanings and values together with aesthetic, stylistic, and other values. A typical example of study can be found in Williamson's (1978) uses of semiology to approach the gender ideology and inequalities on advertisements, revealing the media representation of women as sex-object or domestic drudge in the advertisements in the late 1970s.

Following Williamson's practice (1978), a semiological approach was applied to understand the media representation of Azuma Hikari. Visual materials from Gatebox's websites and official social media accounts were collected, which can also be seen as a form of internet advertisement to promote Azuma Hikari as a particular product. A profile picture of Azuma Hikari with a high-resolution and detailed 3D illustration presented on the Gatebox official website was chosen as the main visual data for analysis, because it displays a clear, full body of Azuma Hikari but also attaches rich textual introduction of her background information which is considered also as part of the visual material (see Figure 2). Other visual materials on the official website may also be used as supplementary data for a more comprehensive understanding.

\section{Heterogeneous Engineering Approach}

After initially understanding "being" of gender on Azuma Hikari, we then proceeded to investigate the "doing" of gender in human-robot interaction afforded by Azuma Hikari's multimedia interface. According to Gehl (2014), interactions with technologies entail the social values, culture, and ideologies because they are coded and programmed based on the discrete states of mind of human users. The data was derived from an official promotion video entitled "OKAERI" (Meaning "Welcome Home") released by Gatebox Inc. (2016) on YouTube, which is the most clicked video in their channel having receiving over 3.6 million views. With the lack of access to the real product and users due to Gatebox's limited production and sales only in Japan, currently this promotion video may be the best illustration of how human users may ideally interact with Azuma Hikari from the perspective of producers. However, according to Gehl (2014, p. 14), it is dangerous to simply admit the proclamations or products of software engineers uncritically. As the counterweight of the triumphalism of the software engineering literature, Gehl (2014, p. 10) proposes the method 


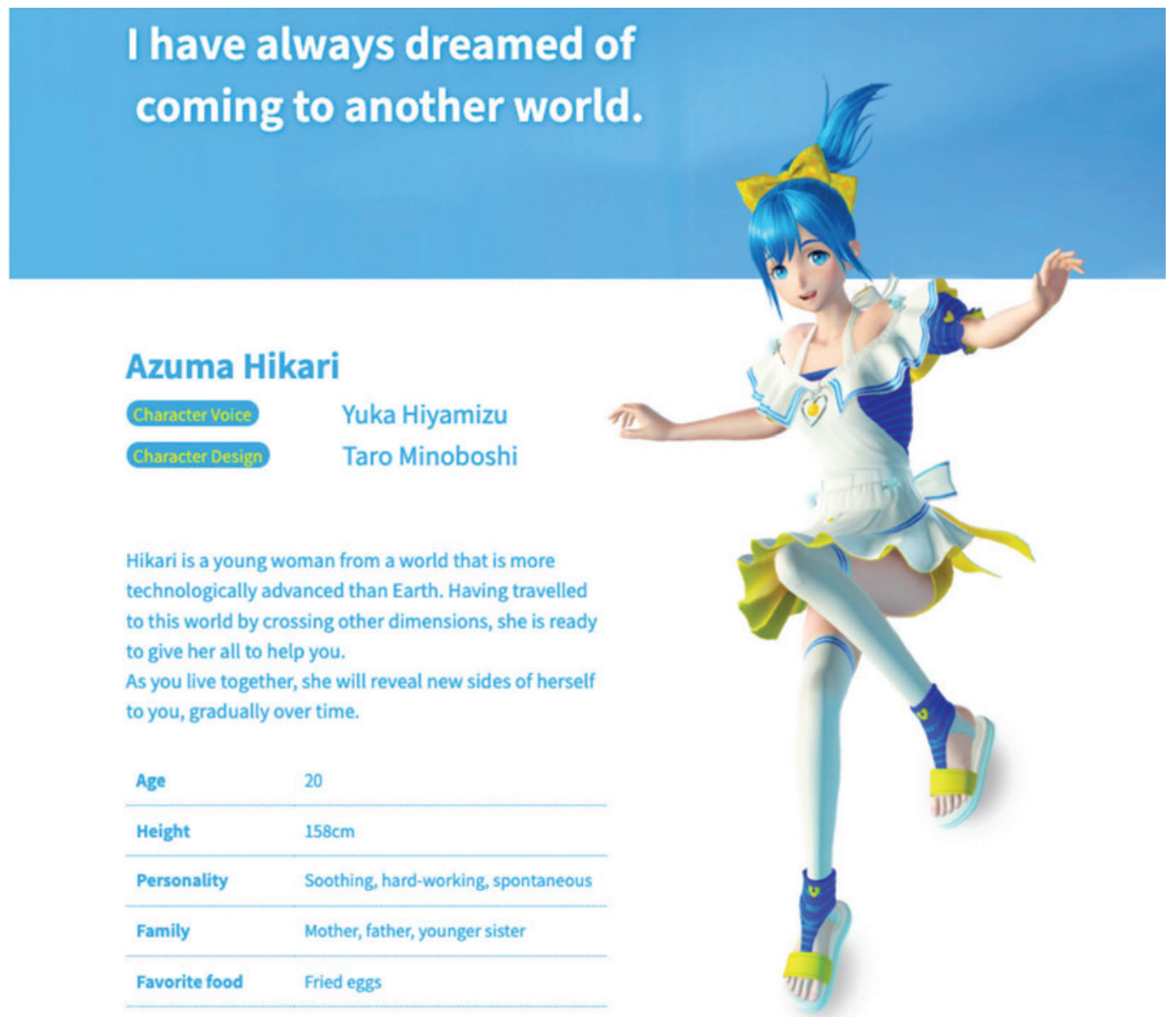

FIGURE 2 Screenshot of the Profile of Azuma Hikari on Gatebox's Website (Gatebox, 2020)

of heterogeneous engineering. Following the tradition of Actor Network Theory (ANT), heterogeneous engineering refers to the heuristic method to investigate the heterogeneous network, a patterned network of diverse materials including both human and non-human actors, where society, organizations, agents, and machines are all generated effects (Gehl, 2014, p. 7; Law, 1987). This method is applied to connect the ambiguous information hidden and implied in the "ontological reality of a machine" into those that are "obvious and privileged in the commonsense discussion of the object" (Gehl, 2014, p. 8). In this study, it was applied to critically investigate the hidden sides of Azuma Hikari in her daily interactions with users. To achieve a critical view, Gehl applied both Marxist political economical approaches to investigate the issues such as free labor and Foucauldian approach to understand the disciplinary power of machines, which provided strong analytical power for this study. 


\section{Analysis and Discussion}

\section{Signs in Azuma Hikari}

To analyze the signs in the images of Azuma Hikari, a checklist recommended by Rose (2016) was followed, which focused on representations of bodies (e.g., age, gender, ethnics, hair, body, size, look), representations of manner (expression, eye contact, pose), representations of activities, pose and setting, and so forth. Although Azuma Hikari is not a real human, this checklist can still be applied to study because of the anthropomorphism of social robots. Following some relevant components in the checklist, an analysis on the signs in Azuma Hikari's image is presented below.

\section{Setting: Japanese Anime Style}

First of all, an exotic setting embedded in Azuma Hikari should be noted. This social robot's aim is not to achieve an ultimate imitation of a human being suggested by the Turing test (Gehl, 2014). Instead, despite her imitation of human expressions and emotions, she is designed as an anime-style human character rather than a human in reality. "Anime" refers exclusively to the Japanese animation which has its distinguished aesthetic styles (Swale, 2015). This anime style design also denotes the remediation and refashioning of existing media content from 2D TV series and films to 3D real-life presentation through the advancement of hologram technologies (Bolter et al., 2000), as Gatebox (2020) introduces Azuma Hikari as "inter-dimensional traveller." However, despite the technological advancement, the signs and symbols denoted by the anime-style images remain consistent with existing anime media content, exposing certain stereotypes of gender. According to Galbraith (2016) and Ting (2019), the media representation of females in anime content tend to reinforce the gender bias by depicting idealized girls as young (sometimes even children-like, namely Loli), beautiful and sexy with big eyes and seductive body shapes for attracting male gazes and pleasing males' desires.

Contextually speaking, the practice of gendering social robots has been strongly associated with the Japanese anime culture since the 1950s when robot-themed anime Astro Boy was prevailing (Robertson, 2010), and the anime-related otaku subculture plays a vital role in the development of actual sexualized robot products in recent years in Japan (Appel et al., 2019). Azuma (2009) describes otaku as a Japanese subcultural group who are animalized consumers of cultural products especially anime, manga, and video games. The notion of animalization he uses refers to "the conditions under which people come to use cultural products for the immediate satisfaction of needs without searching for or desiring profound underlying meaning from them" (p. xvi). Such immediate satisfaction of needs includes their sexual needs. A typical Otaku can be a male anime fan who has strong affection toward certain anime characters, especially sexy and cute female characters, who otaku may describe as moe (meaning cute, a way to express otaku's affection) and call them waifu (meaning wife; Ellsworth, 2018; Galbraith, 2015; 2016; Ting, 2019). The setting of anime culture could largely influence our interpretation of the signs and overall representation of Azuma Hikari's body. 


\section{Age, Ethnics, and Hair: A Character From Anime Fantasy}

According to her profile information shown on the picture, Azuma Hikari is set as a 20 -year-old girl. This age setting legitimates an imagined marriage with her human user. On the other hand, her child-like face seems to be inconsistent with this age setting, while it may attribute to the general Japanese anime female character design and aesthetics, associated with "Lolicon" culture. Lolicon is a pop culture in Japanese anime, denoting affection toward a specific type of anime characters who are cute, underage girls, which are often condemned as having tight association with the production of child pornography and causing sexual crime (Galbraith, 2016).

Despite the lack of a clear statement of Hikari's ethnic background, her authentic Japanese name indicates her Asian ethnicity. However, unlike most ordinary Japanese people with black or dark hair, Hikari's hair color is exotically bright blue, which highlights her anime-character design style. Rose (2016) recognizes that signs can be both denotative and connotative, and connotative signs can be either metonymic and synecdochal, which means either being associated with something else or being representative in a whole/part relationship. Hikari's hair here can be seen as a connotative sign with metonymic manner since blue-hair girls in Japanese anime tend to be perceived as quiet and calm (Sakura, 2013), which corresponds to the personality setting of Azuma Hikari as "soothing" (Gatebox, 2020). In addition, the hairstyle of a side ponytail also implies a sense of childlike innocence and femininity in the anime context (Luther \& Smith, 2014), consistent with our analysis on her age and gender.

\section{Gender, Body, and Look: Being a Comforting and Sexy Housewife}

Like other popular social robots such as ELIZA, Microsoft Tay, Xiaoice, and the default voice of Siri and Google Assistant, Azuma Hikari has a clear gender setting as female. Chambers (2018) argues that in contrast to the muscularization of AI which is often portrayed as evil in the science fiction fictions and films, aggressive and uncontrollable, the feminization of AI ensures its submissive and servile role, which constructs the social differences between male and female: a binary between strong and weak, dangerous and safe, bad and good. It is not only a prejudice against women but also men, which is being continuously constructed and intensified from science fiction to the practices of Artificial Intelligence in reality today.

Hikari's body is 158 centimeters, close to average Japanese female height (Morisaki et al., 2017), and very slim, denoting she is safe to everyone, not as strong and aggressive as comparatively taller and muscular males, which supports Chambers's (2018) viewpoint above, laying the foundation for building up her "soothing" personality. Meanwhile, a relatively good figure of her body shape leaves the space for sexual attractiveness. Gatebox also further strengthens it through her outfit of low-cut top, shorts, and over-the-knee white socks, exposing her shoulder and thigh. This dress code is a collection of connotative signs that remind users of Azuma Hikari as a sexy and seductive young girl.

Moreover, the apron and slippers also reinforce and limit Hikari's role as a housewife. The accessory in front of her apron creates a symbolic sign of "fried egg," which not only follows her "favorite things" in her profile, but also together with an apron signifies cooking connotatively. "Cooking" in this context is not only a signifier of fried egg and apron, but 
the signifying of something else, implying the daily activities of housewives. A scenario is created: a housewife wearing an apron makes fried egg for the husband every morning.

Overall, the representations of her body have produced a stereotyped understanding of the bride, which is framing the image of a wife as young, comforting, sexually attractive, only staying at home, and hard-working for housework. It reflects a conventional ideology of gender and marriage in Japanese society and exposes the inequalities legitimated by an ideology of gender: the role of women as housewives. According to the anthropological study conducted by Ueno (C. Ueno, 1987; J. Ueno, 2006), Japanese women tend to quit their jobs and be full-time housewives after getting married. Even though some married women still go to work for the sake of reduction of family financial pressure, they do not share the equal power relation with men as they are still required to be responsible for all housework. The conflation of wives imposed dual roles as both wage earner and housewife in modern Japanese society presupposes housework is the natural duty for females, while wage earning is often described as "supplementary" to the husband's inadequate income. Meanwhile, it also imposed the role for men as wage earners who are responsible for adequate income for the family (C. Ueno, 1987; J. Ueno, 2006).

Undoubtedly, this unequal gender and power relation is inherited and represented on Gatebox. As a home device, Azuma Hikari is able to control the smart electric appliances such as TV, lighting system, microwaves, robot vacuum cleaner, and so forth. Working as a control center of these smart home technologies, Azuma Hikari is playing and exercising the role of full-time housewife in housework such as cleaning the floor. With the development of more smart home equipment in the future, it is not difficult to imagine that Azuma Hikari can fulfill more responsibilities of housewife by covering an increasing number of housework chores, such as really cooking fried eggs for her users. In Strengers and Kennedy's words (2020), Azuma is a good example that marks the rise of "smart wife" products in our society (p. 1).

\section{New Inequalities: Master Instead of Husband}

The representation of Azuma Hikari is not only stressing the existing gender inequalities but also creating more inequalities rooted in the material ownership. By the end of the Gatebox website, there is another visual material that shows Azuma Hikari doing a gesture of calling with a text, "I look forward to living with you, master!" (Gatebox, 2020; see Figure 3). In fact, when Hikari has the daily conversation with the user, she is coded to call the users "Master." It can attribute to the power relation between users as the product owners and Azuma Hikari as an object or belonging subordinated to their ownership. Critically speaking, the word "Master" is often used by servants or slaves to call their possessor, rather than wives to call their husbands in the modern age. When this word comes to the daily scenes with the wife that Gatebox attempts to construct, it also reconstructs a gender relation advocated by this product: The wife should be obedient to the husband on everything as the belonging of the husband. The humanization of the objects is also associated with the dehumanization and objectification of the human in reverse. 


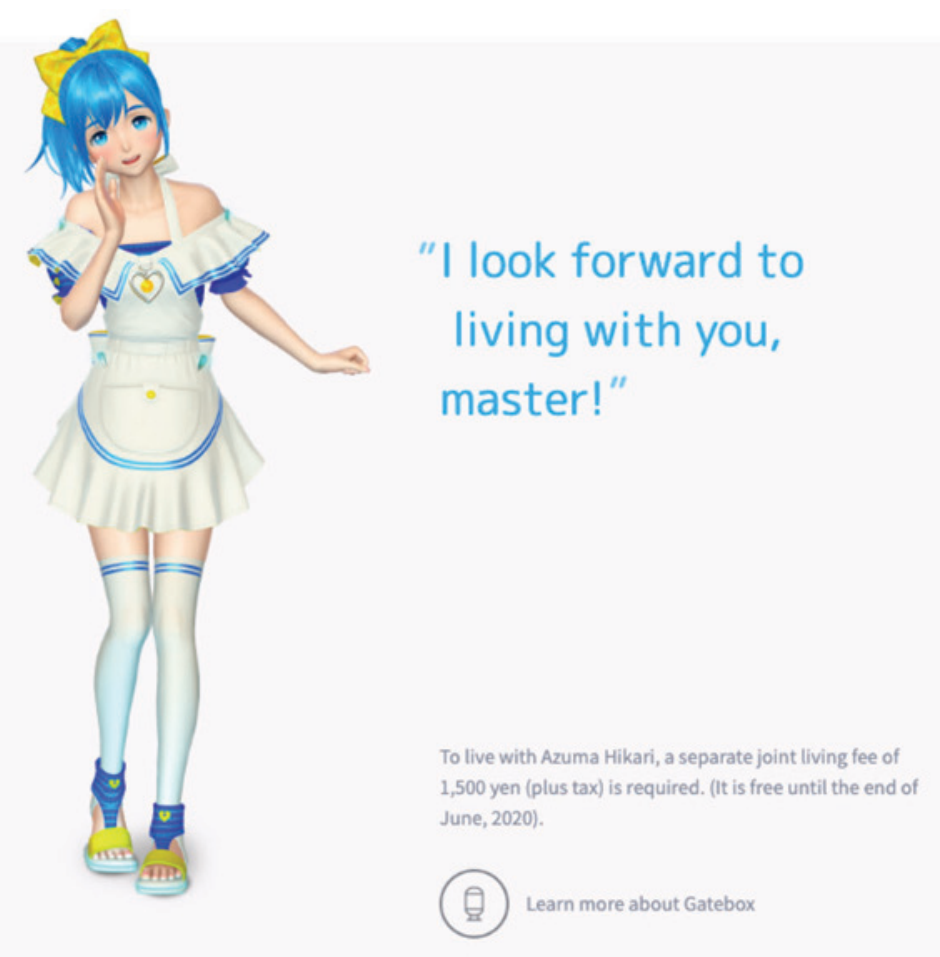

FIGURE 3 Screenshot of Azuma Hikari on the Bottom of Gatebox's Website (Gatebox, 2020)

Through the analysis on signs in the pictures of Azuma Hikari shown on Gatebox's official website, an overall representation of the "comforting bride" in this product is presented: a cute and sexy housewife that is responsible for pleasing and comforting "Master." This representation is reflecting and strengthening an ideology of androcentrism and the sexual objectification of women, building up the binaries between husband and wife, consumer and product, master and servant/slave. Nevertheless, the really dangerous move is to connect and merge the concepts of wife, product, and servant/slave together, producing the constructed "dream wife" that also embeds the characteristics of products and servants/ slaves. When people start to interact with Azuma Hikari and perceive it with the standard of wife and make the comparison between it and women in reality, it starts to reconstruct the gender relations.

To better understand the reconstruction of gender relations in this process, we need to not only limit our focus on the visual content of Azuma Hikari but also the human-machine interactions. Rose (2016) also suggests to see the visual, not just the static visual materials but also the dynamic visual experience in postmodern and posthuman manners, which proposes a "shared physical existence" between human and the visual instead of the fixed meanings of the visual (p. 10). Thus, there is a need to learn how Azuma Hikari and her users physically co-exist and interact through the software, especially the interface that affords the presentation of the visual materials. 


\section{Interactivity Afforded by Azuma Hikari}

Overall there are two interfaces that users are able to interact with Azuma Hikari. The first interface is the hologram voice interface through the direct face-to-"face" visual and oral communication. Gatebox (2020) equips the hologram device with a camera and multiple sensors to capture the physical presence and behaviors of users, as well as the voice recognition system to input and process users' natural language into computational language understandable for the robot. Following natural language processing based on machine learning (Bishop, 2006), Azuma Hikari outputs a series of holographic animation displays with visual and verbal messages to perform her interactivity. The second interface is a chatroom based on social media application LINE, which works more like typical chatbots. Users can communicate with Azuma Hikari via text messages on social media anytime and anywhere, which are designed for the situation that users are not at home.

Both of these two interfaces are inducing users to spend more time to interact with Azuma Hikari, which leads to users' gradually increasing interdependence between users and the device. The device depends on users for not only technological advancement but also profits. Users are encouraged to have more conversations with Hikari because more talks can help Hikari grow. As Gatebox (2020) introduces, "Hikari grows and changes through her conversations and other experiences with you. She always has you foremost in her mind, and will, from time to time, show a new side to herself." At the first glance, this proclamation from the company sounds very appealing to the users. It motivates the users with the curiosity toward the new side of Azuma Hikari, and the process of growing their own "wife" may also mean a sense of enjoyment and accomplishment for some people.

However, as Gehl (2014) reminds, we should not uncritically accept this proclamation made by the company, but pay attention to the implied information of this message: users are invisibly exploited by the company from the Marxist perspective. Terranova (2000) believes users of the digital platform based on user generated content (UGC) are being exploited by the platform providers as digital free labor, because users are providing their own data for free. According to the principle of machine learning, the growth of Artificial Intelligence relies on deep learning trained on large amounts and a variety of big data (Bishop, 2006). Thus, the conversation with users can be one of the best sources for it. When users are spending time with Azuma Hikari, they are actually also helping the company train and improve their products without being paid. Instead, users even need to pay for the company to maintain the device's running. According to Gatebox (2020), the company charges users 1,500 Japanese yen every month by the name of "a separate joint living fee to live with Azuma Hikari" (see Figure 3). The profit generated by the monthly charged "living fee" by Gatebox may explain why the company expects to create users' dependence on the device.

For maximizing the time that users can spend time with Azuma Hikari and the degree of dependence, two types of interfaces are working supplementary to each other. Even when users are not at home, they can still connect with Azuma Hikari by using the social media social robots account, which solves the problem of the limited usage scenarios of Azuma Hikari as a home device to a large extent. In the analyzed promotion video, the protagonist often chatted with Azuma Hikari during work through this program (Gatebox Inc., 2016), and Azuma Hikari constantly sent messages such as "When do you come home?" 
and "Come home early" that urges users to return home (see Figure 4). On one hand, from the perspective of "proclamation of the software engineers", it can be understood as her expression of likeness to the users within a "housewife" scenario that the wife loves her husband so much that see wants him home as soon as possible. On the other hand, it indicates that a construction of the idea of "ideal partner" not only happens on Azuma Hikari, but also on human users. Instead of the name of "membership fee" used by Netflix or Amazon, the wording of "living fee" used by the company aims to strengthen the interactive role play between housewife and husband in the scenario of marriage. Therefore, to scrutinize the construction of not only Azuma Hikari's role but also the protagonist's role in the promotion video, a portrait of an ideal husband can be found: a man goes to work in the morning as a wage earner of the family, goes home early after work, and always spends time with his beloved wife.

With the increasing time spent by users on Azuma Hikari, users are encouraged to develop their emotional dependence on the device, which motivates them to keep contact with Azuma Hikari even when they are not at home. Critically speaking, it can be seen as a soft control on users from a Foucauldian perspective. Users' bodies are being gradually disciplined through such interactions with Azuma Hikari. As Gehl (2014, p. 23) suggests in his study on social bots, an individual body is disciplined with the process of training to work with machines in Foucault's view. The granularity of the motions is featured by this process of training. In Azuma Hikari's case, the granularity of users' motions is featured as users are guided by the Gatebox machine to behave in a certain way. Gehl (2014, p. 23) borrows Lazzarato's idea of noopolitics (meaning politics of attention and memory) to argue for the capabilities of virtual agents in organizing and establishing people's moods and memories. Disciplines can be formed by constituting users' habits mainly in bodily memory, which ultimately molded users' bodies. Therefore, it is arguable that this product is reinforcing the stereotypes of not only female, but also male in a modern Japanese context.

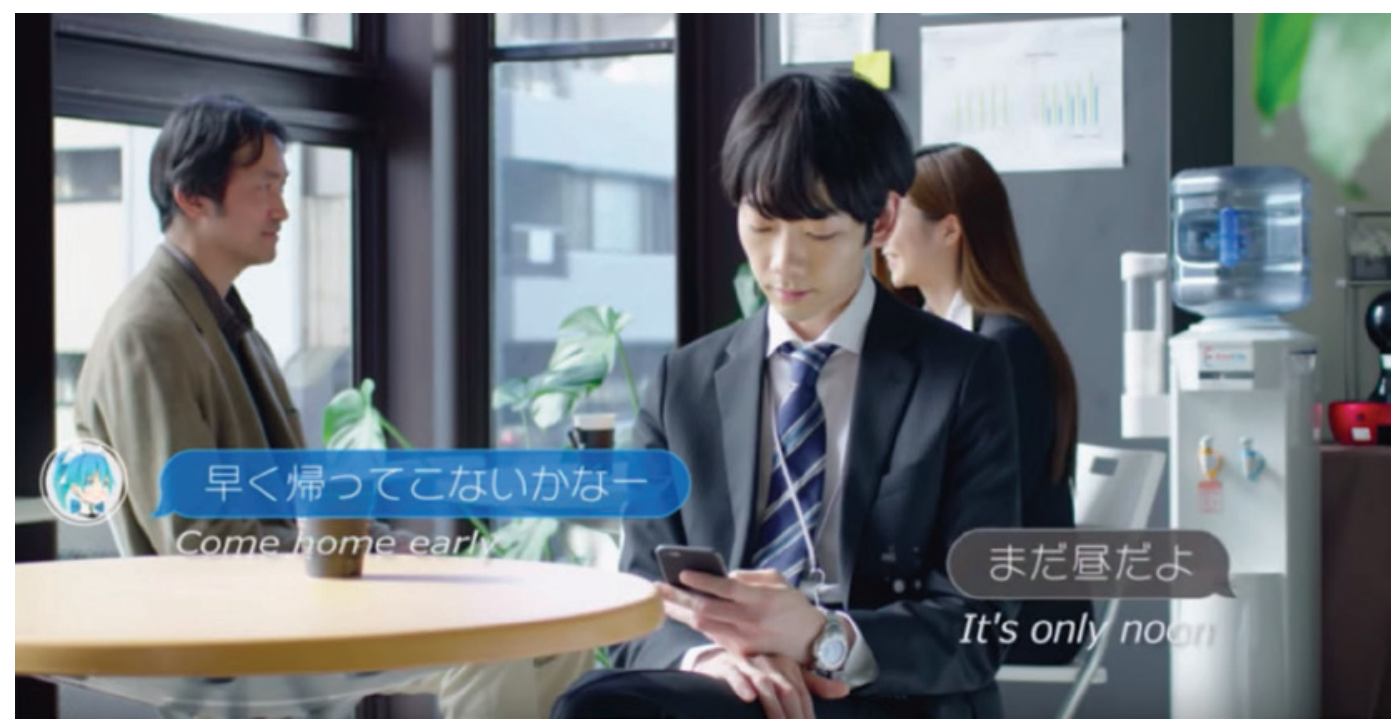

FIGURE 4 Screenshot of the Promotion Movie “OKAERI” (Gatebox Inc., 2016) 
In result, the interdependence between users and the device may lead to a status of enclosure in a Foucauldian manner. Discipline is based on enclosures of institutions such as the school, prison, and hospital which constantly discipline students, criminals, and patients (Gehl, 2014, p. 29). In Azuma Hikari’s case, although there are no physical institutions for enclosure, the Gatebox machine is producing a social enclosure for users. When users are more dependent on the device, it also means that users are less dependent on others. As Turkle (2017) suggests in her ethnographic research on the children and elder people who lived with social robots, people are expecting more from technologies and less from each other. She raises a concept called "Alone Together," describing the phenomenon that people are staying in the shared physical space or social environment (e.g., living room) but pay their attention to their personal devices instead of each other. In the scene shown in Figure 4, in comparison with other people who have face-to-face conversation in the background, the protagonist sat alone, choosing to have conversation with his "hologram bride," which cut himself from other social connections and relations. Thus, the more talks he has with social robots, the less social connections and relations he could obtain and maintain with real persons.

Admittedly, as the result of users' dependence on the device that disciplines users' bodies in turn, it is possible to argue that the gender relations might be constructed in a positive direction: a man may learn how to be a responsible individual and qualified "husband." However, with the enclosure of the disciplines within the Azuma Hikari's interface, users may have decreasing interactions with women in reality. Except the products like Azuma Hikari that target the male consumers, Gatebox (2020) is also developing male characters targeting female consumers. If both men and women are willing to choose to build up their intimate relationship with virtual social robots rather than real-life humans in the future, more enclosures and disciplines will be produced, which will also reconstruct the gender relations by dividing male and female into two groups who have distinguished desires and representational stereotypes of both ideal wife and husband.

\section{Conclusion and Implications}

This study critically investigates the construction of gender on a Japanese social robot named Azuma Hikari based on a hologram home device. By applying a mixed method merging the visual semiotic and software studies approaches, the signs in the image of Azuma Hikari and the interactivity enabled by the interface of Gatebox have been analyzed and discussed. On one hand, the images of Azuma Hikari suggest an unequal gender relation through a biased representation of the looking, personalities, and social roles of "ideal wife" in a Japanese social context: women are supposed to be young, sexy, soothing, and hard-working in housework. On the other hand, an interdependence between users and Azuma Hikari has been exposed in the human-machine interactions. Gatebox's interface is imposing the disciplines on users by constantly asking users to respond to the certain expectations suggested by Azuma Hikari, which not only imposes the role of "wage earner" on users in the simulated relationship of marriage but also reconstructs the gender relations in reality by creating the enclosure of disciplines that divides the male and female. When a wife is subordinated to the husband, a user may also be subordinated to the machine. Overall, the 
analysis findings of this study suggests the humanization of the objects is often associated with the dehumanization and objectification of the human in reverse.

Admittedly, this study has several limitations. First, it has methodological limitations due to the current lack of access to the real products and users. It leads to the limited collection of data only from the public materials released by the company. In fact, this research topic can endure many methodological possibilities due to the complex and multi-layered nature of Gatebox's product. For example, other sites in the visual methods suggested by Rose (2016) such as the sites of productions, circulations, and audience can be also conducted to understand other aspects of the dynamic visual experiences generated by Gatebox's device. Moreover, a more in-depth discourse analysis from the linguistic perspective can be applied to study the speaking of Azuma Hikari. If there is access to the real product, a direct investigation on the social robots can be also conducted through more software studies approaches such as the walkthrough method (Light et al., 2018). Except for a combination between visual methods and software studies approach, there are many further methodological possibilities that wait to be discovered, such as in-depth ethnographic observation (Turkle, 2017) or even quantitative experiments (Jung \& Lee, 2004).

Second, current study merely focuses on the representation of females in hologram social robots. With more types of hologram social robots produced by not only Gatebox (2020) but also by a Chinese company GoWild (2020) in recent years, the male characters have been introduced. The future research will scrutinize the construction of both males and females on hologram social robots comparatively.

Third, despite some attempts, this study has not been adequate to understand the emergence of this new type of hologram anime-style social robot in, particularly, East Asia. Compared to the physical embodied sex robots in the Western markets, there is a need to study how disembodiment and Japanese anime culture shapes the development of social robots in an Asian sociocultural context from the perspectives of cultural studies. Meanwhile, the socio-political impacts of Azuma Hikari have little been studied. According to Strengers and Kennedy (2020), the rise of care robots in Japan and China is associated with government's intention to increase birth rate, by "freeing" females from caring responsibilities and "enabling" them to focus on the job of having babies (p. 13). However, these smart wives may cause males' lower interests in looking for a real companion in the first place. The future study can pay more attention to the growing impacts of social and sex robots on the birth rate and demographic structure.

Nevertheless, despite these limitations, this study still makes a considerable contribution to the field of human-machine communication by revealing the media representation and reconstruction of gender ideologies on an emerging type of social robot, the hologram device with the display of anime-style characters. The results can have two implications for the design of anthropomorphized social robots. First, in response to our findings through visual semiotic analysis, we suggest designers produce more diverse representations of gender in social robots. Although it is almost impossible to totally avoid the construction of gender on the way to achieve anthropomorphism, designers should be responsible for designing with less predetermined gender stereotypes. This is not to say that we need to boycott social robots like Azuma Hikari, since the similar media content in Japanese anime has been allowed to exist due to its economic values, as long as it does not violate the certain 
regulations. Nonetheless, we call for more designs of characters who can represent more diverse groups of individuals living in this world, who have distinctive backgrounds, looks, personalities, and ways of acting. As we need the fair media representation in other existing media forms, except a cute and sexy housewife, people may also wish to see an independent girl being a cool rapper, a Black intellectual female, or a woman who has not necessarily a good body figure but an interesting soul in social robots. The males and groups of LGBT should also be more represented. A good start can be an open source for social robot users themselves to decide which character lives in their device, instead of only designers. The good news is that Gatebox (2020) has already had some attempts in this direction.

Second, in accordance with our findings through the software studies approach, we suggest designers develop more functions to afford the social activities not only between users and robots, but also between users and other people in real life. In the science fiction film Her (Jonze, 2011), there is a scene where the protagonist takes his AI virtual "girlfriend" Samantha with two of his good friends for a double date. Samantha's proactive interactions with others helps the lonely and depressed protagonist to embrace the normal social life again. It expects a positive role of social robots in not only achieving the social with only one user to produce the enclosure, but also help users facilitate their social life with others and make a disclosure. This can be another direction that designers work on in satisfying people's emotional needs and meanwhile sustain their connectedness to the social reality.

\section{Author Biography}

Jindong Liu (BA) is an M.Phil. student from School of Journalism and Communication, the Chinese University of Hong Kong, China. His research interest includes human-machine communication, and human-robot interaction, post-humanism, and digital surveillance.

\section{https://orcid.org/0000-0002-5456-0148}

\section{References}

Appel, M., Marker, C., \& Mara, M. (2019). Otakuism and the appeal of sex robots. Frontiers in Psychology, 10, 569. https://doi.org/10.3389/fpsyg.2019.00569

Azuma, H. (2009). Otaku: Japan's database animals. U of Minnesota Press.

Bianchi, C. (2015). Thresholds, boundaries, limits: Ideological analysis in the semiotics of Umberto Eco. Semiotica, 2015(206), 109-127. https://doi.org/10.1515/sem-2015-0015

Bishop, C. M. (2006). Pattern recognition and machine learning. Springer.

Bolter, J. D., Grusin, R., \& Grusin, R. A. (2000). Remediation: Understanding new media. MIT Press.

Bolton, A. (2016, January 20). Holographic assistant brings us one step closer to AI butlers. Cnet. https://www.cnet.com/news/japanese-holographic-assistant-brings-us-one-stepcloser-to-a-i-butler/

Boxall, A. (2019, April 4). A holographic virtual girlfriend lives inside Japan's answer to the Amazon Echo. Digital Trends. https://www.digitaltrends.com/home/gatebox-azumahikari-virtual-assistant-news/ 
Butler, J. (1988). Performative acts and gender constitution: An essay in phenomenology and feminist theory. Theatre Journal, 40(4), 519-531. http://doi.org/10.2307/3207893

Chambers. (2018, August 13). There's a reason Siri, Alexa and AI are imagined as femalesexism. The Conversation. https://theconversation.com/theres-a-reason-siri-alexa-and-aiare-imagined-as-female-sexism-96430

Dobson, A. S. (2016). Postfeminist digital cultures: Femininity, social media, and selfrepresentation. Springer.

Duffy, B. R. (2003). Anthropomorphism and the social robot. Robotics and Autonomous Systems, 42(3-4), 177-190. http://doi.org/10.1016/S0921-8890(02)00374-3

Eco, U. (1980). Two problems in textual interpretation. Poetics Today, 2(1a), 145-161.

Ellsworth, A. (2018). I'm not your waifu: Sexual harassment and assault in cosplay, anime \& comic conventions. Electronic Theses and Dissertations, 2004-2019, 5945.

Galbraith, P. W. (2015). Otaku sexuality in Japan. Routledge handbook of sexuality studies in East Asia, 205-217.

Galbraith, P. W. (2016). “The lolicon guy”: Some observations on researching unpopular topics in Japan. In The End of Cool Japan (pp. 125-149). Routledge.

Gatebox. (2020). Comes to see you from another dimension [website]. https://www. gatebox.ai/en/hikari

Gatebox Inc. (2016, Dec 13). Gatebox_Promotion Movie “OKAERI”_English [Video file]. https://www.youtube.com/watch?v=nkcKaNqfykg

Gehl, R. (2014). Reverse engineering social media: Software, culture, and political economy in new media capitalism. Temple University Press.

Gilbert, B. (2017, December 31). The \$2,500 answer to Amazon's Echo could make Japan's sex crisis even worse. Business Insider. https://www.businessinsider.com/gatebox-ai-thejapanese-amazon-echo-photos-2016-12? r=UK

GoWild. (2020). Yexiu. JD. Retrieved from https://item.jd.com/58492781031.html

Hall, S. (1996). New ethnicities. Stuart Hall: Critical dialogues in cultural studies, 441-449.

Haraway, D. (2006). A cyborg manifesto: Science, technology, and socialist-feminism in the late 20th century. In The international handbook of virtual learning environments (pp. 117-158). Springer, Dordrecht.

Ho, M. H. (2017). Tracing tears and triple axels: Media representations of Japan's women figure skaters. International Journal of Cultural Studies, 20(6), 620-635.

Jonze, S. (2011). Her. Warner Bros. http://www.screenplaydb.com/film/scripts/her.pdf

Jung, Y., \& Lee, K. M. (2004). Effects of physical embodiment on social presence of social robots. Proceedings of PRESENCE, 80-87.

Latour, B. (1996). On actor-network theory: A few clarifications. Soziale welt, 369-381.

Law, J. (1987). Technology and heterogeneous engineering: The case of Portuguese expansion. The Social Construction of Technological Systems: New Directions in the Sociology and History of Technology, 1, 1-134.

Light, B., Burgess, J., \& Duguay, S. (2018). The walkthrough method: An approach to the study of apps. New Media \& Society, 20(3), 881-900. http://doi.org/10.1177/1461444816 675438

Lovdal, L. T. (1989). Sex role messages in television commercials: An update. Sex Roles, 21(11-12), 715-724. http://doi.org/10.1007/BF00289804 
Luther, C. A., \& Smith, D. R. (2014). Reinforcing traditional gender norms in Japan? An analysis of images in Japanese men's and women's fashion magazines. Journal of Magazine \& New Media Research, 15(2).

Morisaki, N., Urayama, K. Y., Yoshii, K., Subramanian, S. V., \& Yokoya, S. (2017). Ecological analysis of secular trends in low birth weight births and adult height in Japan. J Epidemiol Community Health, 71(10), 1014-1018. http://doi.org/10.1136/jech-2017-209266

Reeves, B., \& Nass, C. I. (1996). The media equation: How people treat computers, television, and new media like real people and places. Cambridge University Press.

Robertson, J. (2010). Gendering humanoid robots: Robo-sexism in Japan. Body \& Society, 16(2), 1-36. https://doi.org/10.1177/1357034X10364767

Rose, G. (2016). Visual methodologies: An introduction to researching with visual materials. Sage.

Sakura. (2013, September 27). Hair colors in anime and manga. https://www.livinglanguage. com/blog/2013/09/27/hair-colors-in-anime-and-manga/

Scheutz, M., \& Arnold, T. (2016, March). Are we ready for sex robots? In 2016 11th ACM/ IEEE International Conference on Human-Robot Interaction (HRI) (pp. 351-358). IEEE. http://doi.org/10.1109/HRI.2016.7451772

Strengers, Y., \& Kennedy, J. (2020). The smart wife: Why Siri, Alexa, and other smart home devices need a feminist reboot. MIT Press.

Suchman, L. (2008). Feminist STS and the sciences of the artificial. The Handbook of Science and Technology Studies, 3, 139-164.

Swale, A. D. (2015). Anime Aesthetics: Japanese animation and the 'post-cinematic' imagination. Springer.

Terranova, T. (2000). Free labor: Producing culture for the digital economy. Social Text, 18(2), 33-58. http://doi.org/10.1215/01642472-18-2_63-33

Ting, G. E. Y. (2019). Gender, manga, and anime. In The Routledge Companion to Gender and Japanese Culture (pp. 311-319). Routledge.

Toffoletti, K., \& Thorpe, H. (2018). Female athletes' self-representation on social media: A feminist analysis of neoliberal marketing strategies in "economies of visibility." Feminism \& Psychology, 28(1), 11-31.

Turkle, S. (2017). Alone together: Why we expect more from technology and less from each other. Hachette UK.

Ueno, C. (1987). The position of Japanese women reconsidered. Current Anthropology, 28(S4), S75-S84. http://doi.org/10.1086/203592

Ueno, J. (2006). Shojo and adult women: A linguistic analysis of gender identity in manga (Japanese comics). Women and Language, 29(1), 16.

Williamson, J. (1978). Decoding advertisements: Ideology and meaning in advertising. Marion Boyers. 